\title{
Nucleation of superconductivity in finite anisotropic superconductors and the evolution of surface superconductivity toward the bulk mixed state
}

\author{
V. G. Kogan and J. R. Clem \\ Ames Laboratory - DOE and Department of Physics and Astronomy, Iowa State University, Ames, Iowa 50011-3160
}

J. M. Deang

Department of Mathematics, Virginia Tech, Blacksburg, Virginia 24061-0123

M. D. Gunzburger

Department of Mathematics, Iowa State University, Ames, Iowa 50011-2064

(Received 19 September 2001; published 14 February 2002)

\begin{abstract}
In anisotropic superconductors having an arbitrary orientation of the sample surface relative to the crystal principal axes, the surface critical field $H_{c 3}$ is less than $1.695 H_{c 2}$ unless the field is situated along one of the principal crystal planes. Below $H_{c 3}$ in the vicinity of nucleation, the order parameter scales as $\sqrt{H_{c 3}-H}$. Computational studies for infinite cylinders having rectangular cross sections are presented which show that, due to corners and a finite cross section, the surface superconductivity state persists for fields above the theoretically predicted value for semi-infinite samples. They also show that vortices exist within the surface superconductivity sheath above the bulk critical field.
\end{abstract}

DOI: 10.1103/PhysRevB.65.094514

PACS number(s): 74.20.De, 74.25.Ha, 74.60.-w

\section{INTRODUCTION}

The maximum field, $H_{c 3}$, at which superconductivity first nucleates at the surface of isotropic materials, is found by solving the linearized Ginzburg-Landau (GL) equation for the order parameter $\psi$,

$$
-\xi^{2} \Pi^{2} \psi=\psi
$$

in a half-space (say, $x>0$ ) with the GL boundary condition $\Pi_{x} \psi=0$ at the surface and $\psi(x \rightarrow \infty)=0$. Here, $\xi$ is the coherence length and $\boldsymbol{\Pi}=\nabla+2 \pi i \mathbf{A} / \phi_{0}$ with $\mathbf{A}$ and $\phi_{0}$ the vector potential and the flux quantum, respectively. The problem is uniform in the $y$ and $z$ directions, and $\nabla$ has only one component, $d / d x$. The maximum value $H_{c 3}$ is achieved when the field is parallel to the surface; thus, we choose the applied field $\mathbf{H}=H \hat{\mathbf{z}}$. Since $f \equiv|\psi|$ is infinitesimal at the nucleation point, the field is uniform, and one can take the Landau gauge for the vector potential: $A_{y}=H x, A_{x}=A_{z}=0$. Looking for a solution to Eq. (1) of the form $\psi=f(x) \exp$ (-iky), one obtains

$$
f^{\prime \prime}-q^{4}\left(x-x_{0}\right)^{2} f=-f / \xi^{2},
$$

where $q^{2}=2 \pi H / \phi_{0}$ and $x_{0}=k / q^{2}$. The boundary conditions are $f^{\prime}(0)=0$ and $f(\infty)=0$. Therefore, the problem is reduced to the determination of a maximum value for $q^{2}$ (the field) for the given eigenvalue $\xi^{-2}$; this is achieved by varying $x_{0}$ which is still a free parameter. Saint-James and de Gennes ${ }^{1}$ obtained the value

$$
q^{2}=1.695 / \xi^{2}
$$

i.e., $H_{c 3}=1.695 H_{c 2}$.

Since the work of Saint-James and de Gennes, ${ }^{1}$ there have been a number of theoretical (e.g., Refs. 2-13), experimental (e.g., Refs. 14-16), and computational (Refs. 17-20) studies of the onset of superconductivity in high fields. These studies dealt with isotropic materials and mostly focused on determining the field value at which superconductivity first nucleates in domains other than the half plane considered by Saint-James and de Gennes. In particular, for samples having smooth boundaries, it was found (e.g., see Ref. 8) that superconductivity first nucleates at points on the boundary having maximum curvature and that $H_{c 3}=1.695 H_{c 2} /\left(1-K C_{\text {max }}\right)$, where $C_{\max }$ denotes the maximum curvature of the boundary and $K$ is a constant whose value is material dependent. Thus, the surface nucleation field for samples having smooth boundaries with nonzero curvature is higher than it is for the half plane. Starting with Ref. 3, a number of studies were devoted to domains with corners; again, it was found that the surface nucleation field was higher than for the half plane. Perhaps the results that are most relevant to our study are found in Ref. 12 and in the computational simulations of Refs. 17 and 18, in which it was determined that for isotropic material samples with square corners, e.g., the quarter plane, $H_{c 3} / H_{c 2}$ has a value between 1.8 and 2 . Note that this is higher than 1.695, the value found by Saint-James and de Gennes for the half plane.

Previous studies of the surface nucleation field in superconductors not only dealt with isotropic superconductors, but also failed to examine the transition between the vortex state in type-II superconductors for lower fields, say below $H_{c 2}$, and the surface superconducting state near $H_{c 3}$. Thus, in this paper, we first give a theoretical generalization of the SaintJames and de Gennes result to the case of anisotropic superconductors. Then, using computational simulations for the fully nonlinear Ginzburg-Landau equations, the transition between the vortex and surface superconductivity states is examined for both isotropic and anisotropic superconductors. The computational simulations are also used to illustrate the extensions of the Saint-James and de Gennes theory given in Secs. II and III. 


\section{ANISOTROPIC MATERIALS}

For anisotropic materials, Eq. (1) is generalized to

$$
-\xi^{2} \mu_{i j} \Pi_{i} \Pi_{j} \psi=\psi
$$

Here $\mu_{i j}=m_{i j}^{-1}$ is the inverse mass tensor. The tensor of "superconducting masses" $m_{i j}$ has in general three different eigenvalues $m_{a}, m_{b}$, and $m_{c}$; these are commonly normalized so that $m_{a} m_{b} m_{c}=1$. The average coherence distance is defined as $\xi=\left(\xi_{a} \xi_{b} \xi_{c}\right)^{1 / 3}$, where the actual coherence lengths are $\xi_{i}=\xi / \sqrt{m_{i}}$. The GL boundary condition imposed on the free surface is now given by

$$
\mu_{x j} \Pi_{j} \psi=0,
$$

where the $x$ direction is perpendicular to the surface.

Let us introduce the modulus and phase of the order parameter, $\psi=f e^{i \chi}$, so that $\Pi \psi=e^{i \chi}(\nabla f+i \mathbf{Q} f)$, where

$$
\mathbf{Q}=\boldsymbol{\nabla} \chi+2 \pi \mathbf{A} / \phi_{0} .
$$

In terms of these variables, Eq. (4) contains the factor $e^{i \chi}$ on both sides. After canceling this factor and separating the real and imaginary parts, one obtains

$$
\begin{gathered}
-\xi^{2} \mu_{i k}\left(\frac{\partial^{2} f}{\partial x_{i} \partial x_{k}}-f Q_{i} Q_{k}\right)=f, \\
\mu_{i k}\left[\frac{\partial f}{\partial x_{k}} Q_{i}+\frac{\partial}{\partial x_{i}}\left(f Q_{k}\right)\right]=0 .
\end{gathered}
$$

One easily verifies that Eq. (8) coincides with $\operatorname{div} \mathbf{j}=0$, where the current density $j_{i} \propto f^{2} \mu_{i k} Q_{k}$ (the second GL equation). Therefore, we have to solve Eq. (7); for $f=f(x)$ and $\chi=-k_{y} y-k_{z} z$ (in the anisotropic case, currents may have a component along the field direction $z$ as well as along $y$ ) Eq. (7) assumes the form

$$
\mu_{x x} f^{\prime \prime}-\mu_{\alpha \beta} Q_{\alpha} Q_{\beta} f=-f / \xi^{2},
$$

where $\alpha, \beta=y, z$ and $\mathbf{Q}=\left\{0, q^{2} x-k_{y},-k_{z}\right\}$. This equation is of the same type as Eq. (2) for the isotropic case since the coefficient of $f$ is a quadratic polynomial in $x$, i.e.,

$$
\mu_{x x} f^{\prime \prime}-f\left(\mu_{y y} q^{4} x^{2}-2 q^{2} \mu_{y \alpha} k_{\alpha} x+\mu_{\alpha \beta} k_{\alpha} k_{\beta}\right)=-f / \xi^{2}
$$

or

$$
f^{\prime \prime}-\frac{\mu_{y y}}{\mu_{x x}} q^{4}\left(x-x_{0}\right)^{2} f=-\frac{f}{\xi^{2} \mu_{x x}}\left(1-k_{z}^{2} \xi^{2} \frac{d}{\mu_{y y}}\right)
$$

with $x_{0}=\mu_{y \alpha} k_{\alpha} / \mu_{y y} q^{2}$ and $d=\operatorname{det} \mu_{\alpha \beta}$ is the minor $x x$ of $\mu_{i j}$.

The complex-valued boundary condition (5) yields

$$
\mu_{x x} f^{\prime}=0, \quad \mu_{x y} Q_{y} f=0,
$$

i.e., both $f^{\prime}$ and $j_{x}$ vanish at the surface, as expected. Equation (11) differs from its isotropic analog Eq. (2) only in the values of the constant coefficients, so we can utilize the result (3) to obtain

$$
q^{2}=\frac{1.695}{\xi^{2} \sqrt{\mu_{x x} \mu_{y y}}}\left(1-k_{z}^{2} \xi^{2} \frac{d}{\mu_{y y}}\right) .
$$

Straightforward algebra yields that $d=\mu_{y y} \mu_{z z}-\mu_{y z}^{2}>0$ so that the maximum value of $q^{2}$ is achieved for $k_{z}=0$. Thus,

$$
H_{c 3, z}=\frac{1.695 \phi_{0}}{2 \pi \xi^{2} \sqrt{\mu_{x x} \mu_{y y}}} .
$$

Recall that the bulk upper critical field in the direction $z$ is given by (see Refs. 22 and 23)

$$
H_{c 2, z}=\frac{\phi_{0}}{2 \pi \xi^{2} \sqrt{m_{z z}}}=\frac{\overline{H_{c 2}}}{\sqrt{m_{z z}}},
$$

where

$$
\bar{H}_{c 2}=\frac{\phi_{0}}{2 \pi \xi^{2}}, \quad \xi=\left(\xi_{a} \xi_{b} \xi_{c}\right)^{1 / 3} .
$$

Then, since $m_{z z}=\mu_{z z}^{-1}=\mu_{x x} \mu_{y y}-\mu_{x y}^{2} \quad\left(\operatorname{det} \mu_{i j}=1\right)$, we obtain

$$
\frac{H_{c 3, z}}{H_{c 2, z}}=1.695 \sqrt{1-\frac{\mu_{x y}^{2}}{\mu_{x x} \mu_{y y}}} .
$$

Equations (14) and (17) are our main results. Equation (17) shows that if the direction $x$ of the normal to the crystal surface coincides with one of the principal crystal axes (or equivalently, the surface is one of the principal crystal planes), then $\mu_{x y}=0$ and Eq. (17) implies that the ratio $H_{c 3, z} / H_{c 2, z}$ is the same as for the isotropic situation. In other words, in this case the angular dependence of $H_{c 3}$ is the same as that of $H_{c 2}$.

For all other surface orientations, the ratio is less than 1.695. Moreover, if $\mu_{x x} \mu_{y y}-\mu_{x y}^{2} \leqslant 1.695^{-2} \mu_{x x} \mu_{y y}$, or equivalently,

$$
0.652 \mu_{x x} \mu_{y y} \leqslant \mu_{x y}^{2}
$$

$H_{c 3, z} / H_{c 2, z} \leqslant 1$ which means that surface superconductivity is completely suppressed.

As an example, consider the case when the field is applied in a principal crystal direction, say along $a \quad(z=a)$, whereas the axis $b$ forms an angle $\theta$ with the $x$ axis (see Fig. 1). The frame $a b c$ is rotated relative to $x y z$ about the axis $z=a$ :

$$
x=b \cos \theta-c \sin \theta, \quad y=b \sin \theta+c \cos \theta, \quad z=a .
$$

This yields

$$
\begin{aligned}
& \mu_{x x}=\mu_{b} \cos ^{2} \theta+\mu_{c} \sin ^{2} \theta, \\
& \mu_{y y}=\mu_{b} \sin ^{2} \theta+\mu_{c} \cos ^{2} \theta, \\
& \mu_{x y}=\left(\mu_{b}-\mu_{c}\right) \sin \theta \cos \theta .
\end{aligned}
$$

Then, we have that $\mu_{x x} \mu_{y y}=\mu_{b} \mu_{c}+\left(\mu_{b}-\mu_{c}\right)^{2} \cos ^{2} \theta \sin ^{2} \theta$ and, introducing $\gamma_{c b}^{2}=m_{c} / m_{b}=\mu_{b} / \mu_{c}$, we obtain from Eq. (18) 


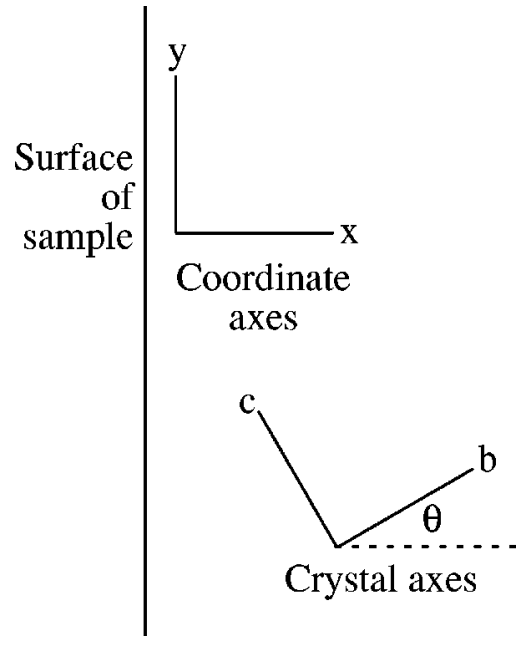

FIG. 1. Sample surface, crystal axes, and coordinate axes for semi-infinite anisotropic sample; the $z$ and $a$ axes are perpendicular to the $b-c$ and $x-y$ planes.

$$
H_{c 3, a}=\frac{1.695}{\sqrt{1+\left(\gamma_{c b}-\gamma_{c b}^{-1}\right)^{2} \cos ^{2} \theta \sin ^{2} \theta}} H_{c 2, a} .
$$

Now, Eq. (20) yields the critical misalignment angle $\theta_{0}$ between the normal to the surface and a principal crystal direction such that for $\theta_{0}<\theta<\left(\pi / 2-\theta_{0}\right)$, surface superconductivity is completely suppressed; this critical angle is determined from

$$
\sin 2 \theta_{0}=\frac{2.737}{\left|\gamma_{c b}-\gamma_{c b}^{-1}\right|} .
$$

For example, for (nearly uniaxial) $\mathrm{Y}-\mathrm{Ba}-\mathrm{Cu}-\mathrm{O}$ with $\gamma \approx 7.9$, $\theta_{0} \approx 11.5^{\circ}$, while for $\mathrm{NbSe}_{2}, \gamma \approx 3.1$ and $\theta_{0} \approx 44.6^{\circ}$. One can see that for $\gamma<3.064, H_{c 3, a}>H_{c 2, a}$ for all values of $\theta$.

\section{BEHAVIOR FOR FIELDS BELOW $H_{c 3}$}

It is of interest to see how the surface state with an infinitesimally small order parameter at $H_{c 3}$ evolves when the applied field decreases. One can obtain some insight by employing the method used to investigate bulk superconductivity in fields under $H_{c 2}$ (see, e.g., Ref. 21).

To find the nucleation field, the linear Eqs. (1) or (4) are solved. These, of course, yield $\psi$ up to a constant factor. To determine this factor, one turns to the full nonlinear GL equation

$$
-\xi^{2} \Pi^{2} \psi=\psi\left(1-\left|\psi^{2}\right|\right)
$$

( $\psi$ is normalized on the zero-field value of the order parameter). For our purpose, it is convenient to write Eq. (22) in terms of the operators $\Pi^{ \pm}=\Pi_{x} \pm i \Pi_{y}$ that have the property $\Pi^{+} \Pi^{-}=\Pi^{2}+q^{2}$; one then obtains

$$
\left(\Pi^{+} \Pi^{-}-q^{2}+\xi^{-2}\right) \psi=\psi|\psi|^{2} / \xi^{2} .
$$

At $H_{c 3}$,

$$
\left(\Pi_{0}^{+} \Pi_{0}^{-}-q_{c 3}^{2}+\xi^{-2}\right) \psi_{0}=0
$$

where $\psi_{0}$ is the order parameter at the point of nucleation and $\Pi_{0}^{ \pm}$correspond to $H_{c 3}$. For a field $H$ slightly below $H_{c 3}$, we look for a solution to Eq. (23) in the form $\psi=\psi_{0}+\psi_{1}$ with $\psi_{1} \ll \psi_{0}$. We now write $q^{2}$ in Eq. (23) as $q_{c 3}^{2}-\left(q_{c 3}^{2}\right.$ $\left.-q^{2}\right)=q_{c 3}^{2}-\delta q^{2}$, and $\Pi^{ \pm}=\Pi_{0}^{ \pm} \pm i \mathbf{a}^{ \pm}$, where $\mathbf{a}$ is a correction to $2 \pi \mathbf{A}_{0} / \phi_{0}$. Substituting in Eq. (23) and keeping only the first-order terms, one obtains for $\psi_{1}$

$$
\begin{gathered}
\left(\Pi_{0}^{+} \Pi_{0}^{-}-q_{c 3}^{2}+\xi^{-2}\right) \psi_{1}= \\
\psi_{0}\left|\psi_{0}\right|^{2} \xi^{-2}-2 \delta q^{2} \psi_{0} \\
-2 i \mathbf{a} \cdot \Pi_{0} \psi_{0} .
\end{gathered}
$$

The operator at the left-hand side of this inhomogeneous equation coincides with that of Eq. (24). Therefore, the righthand side of this equation must be orthogonal to the solution of the homogeneous Eq. (24), i.e., to $\psi_{0}$. Then,

$$
\int_{0}^{\infty}\left(\left|\psi_{0}\right|^{4}-2 \frac{H_{c 3}-H}{H_{c 2}}\left|\psi_{0}\right|^{2}-2 \xi^{2} \mathbf{a} \cdot\left|\psi_{0}\right|^{2} \mathbf{Q}_{0}\right) d x=0 \text {. }
$$

This is the normalization condition for $\psi_{0}$. Since $|\mathbf{a}| \propto\left(H_{c 3}\right.$ $-H)$, the square of the order parameter in the surface sheath should scale with $\left(H_{c 3}-H\right)$. In other words, starting from zero at $H_{c 3}$, the order parameter grows with decreasing field approximately as $\sqrt{H_{c 3}-H}$.

\section{COMPUTATIONAL STUDY OF ISOTROPIC MATERIALS}

The results given so far for surface nucleation at high fields are derived assuming a semi-infinite sample, e.g., the surface of the sample is the plane $x=0$. We now study the effects of anisotropies on surface nucleation at high fields in infinite cylinders having rectangular cross sections through the use of computational simulations. We also examine what happens to the vortex state for fields above $H_{c 2}$ (the critical field for bulk samples), a behavior that cannot be modeled by the semi-infinite, one-dimensional situation of the theoretical developments in Secs. I-III.

For the computational simulations, we use a finite element discretization of the fully nonlinear GL equation, i.e., Eq. (1) for the isotropic case of this section and Eq. (4) for the anisotropic case of Sec. V; details are given in, e.g., Refs. 24 and 25. The sizes of the samples used in the computational simulations are very small, even smaller than were previously used (see, e.g., Refs. 24-26) for fields well below $H_{c 2}$. The reason for this is that the grids used for simulations at fields larger than $\mathrm{H}_{c 2}$ need to be much finer than those for lower values of the field. As we shall see, our computations show that vortices exist within the surface superconductivity sheath. They appear in regions in which the order parameter is already very small in magnitude. The need to differentiate between true zeros of the order parameter and merely small values requires very fine grids. In fact, in a previous computational study ${ }^{17}$ of the surface superconductivity state at high fields, vortices within the surface sheath were not noticed, probably because only about two grid points per coherence length were used. Even so, in our computational simulations, it is possible that we have not captured all vortices that are 


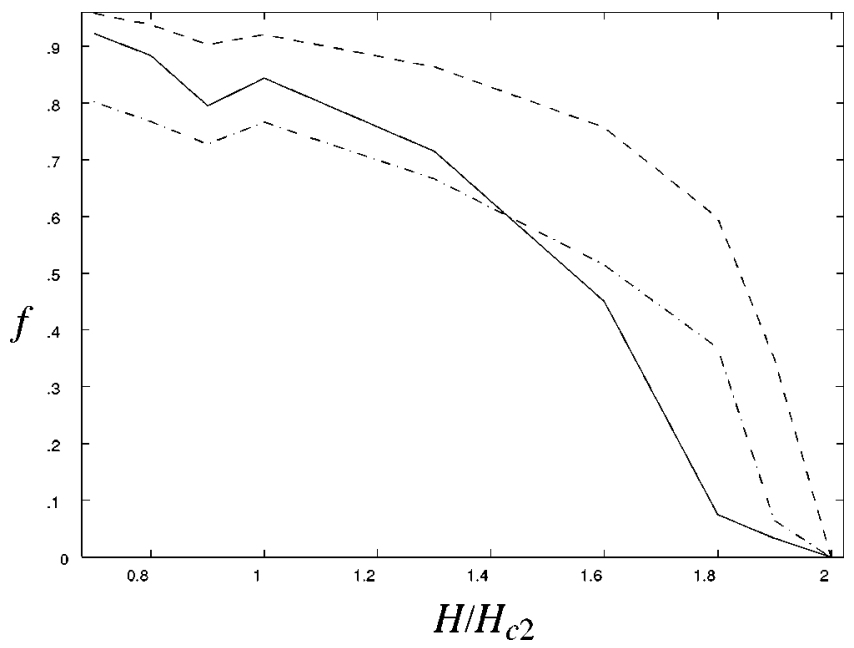

present, especially near the center of the samples where the magnitude of the order parameter is extremely small.

The particular finite element method utilized involves a subdivision of the rectangular cross section of a cylindrical sample into a grid consisting of small squares. Typically, we use up ten points per coherence length; i.e., for a sample of cross-sectional size $10 \xi \times 10 \xi$, we would use up to a 100 $\times 100$ grid. Each square is subdivided into two triangles by introducing a diagonal to effect a triangulation of the cross section. Solutions of the GL equation are then approximated by a continuous, piecewise quadratic polynomial with respect to the triangulation; thus, the discretization method is of third-order accuracy with respect to the grid size. See Refs. 24 and 25 for further details. We merely note that the combination of the use of at least ten grid points per coherence length and the use of quadratic polynomial approximations results in very accurate computational simulations.

We first examine an isotropic, infinite cylinder having a square cross section of size $10 \xi \times 10 \xi$, where $\xi$ is the coherence length. The magnetic field in the calculations ranges from a low field of $0.7 H_{c 2}$ to a field where superconductivity is totally destroyed which, for this sample, is observed to occur at $H_{c 3} \approx 2.0 H_{c 2}$. The magnitude of the order parameter $f \equiv|\psi|$ vs the applied field strength is given in Fig. 2. The solid curve corresponds to $f$ at the midsides, the dashed curve to $f$ at the corners, and the dot-dashed curve to the average of $f$ over the sample perimeter. The curves for $f$ roughly display the square-root-type behavior vs $\left(H_{c 3}-H\right)$ predicted by the theory (see Sec. III). As the field approaches $H_{c 3}$, the average and the corner values of $f$ exceed its value at the midsides of the sample. This is due to corner effects that render the order parameter bigger in the corners than at the rest of the sample in high applied fields near $H_{c 3}$ as discussed in, e.g., Refs. 3, 12, 17, and 18. The notch near $H=H_{c 2}$ is characteristic of many of our results; its meaning is unclear to us at the moment.

A surface plot of $f$ for $H=1.6 H_{c 2}$ is given in Fig. 3. We can clearly see that the order parameter is larger at the corners of the sample than at the midsides. We also see an indication that the state is not merely a surface superconductivity state, but that it also has vortices in the interior of the sample. This is confirmed by an examination of the numeri- cal results which show that the minima exhibited in Fig. 3 correspond to zeros of the order parameter and to phase changes of $2 \pi$, i.e., the minima correspond to vortices. Note that the vortices occur in regions where the order parameter is small in magnitude.

In Fig. 4, contour plots of $f$ are shown for the steady states obtained with the applied magnetic field ranging from $0.7 H_{c 2}$ to $1.8 H_{c 2}$. The first contour corresponds to a field strength of $0.7 H_{c 2}$ which is well below $H_{c 2}$; eight vortices are seen for this small material sample. At $H_{c 2}$, there are eight vortex cores evident near the center of the sample and there are four other vortices appearing nearer to the corners. Then, as the field increases further, the order parameter at the midsides of the boundary and at the center of the sample approaches zero with the corner values lagging behind until the whole sample becomes normal at $H_{c 3} \approx 2.0 H_{c 2}$. This value for $H_{c 3}$ agrees with that found in Ref. 17. One sees the surface superconductivity state appearing for fields between $H_{c 2}$ and $H_{c 3}$. However, note that four vortices persist even at fields between $H_{c 2}$ and $H_{c 3}$. The four vortices coexisting with the surface superconductivity state are important to note since they are always present in the steady state of every isotropic and anisotropic sample we have studied in fields

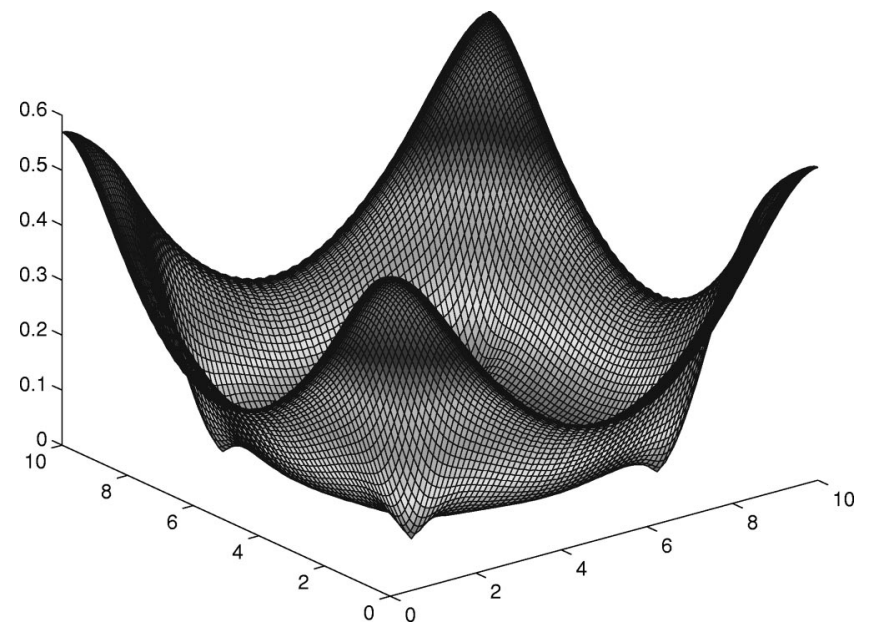

FIG. 3. Surface plot of $f \equiv|\psi|$ for an isotropic sample having a $10 \xi \times 10 \xi$ cross section with field strength of $1.6 H_{c 2}$. 


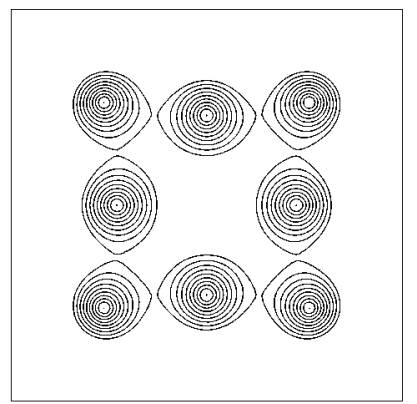

(a)

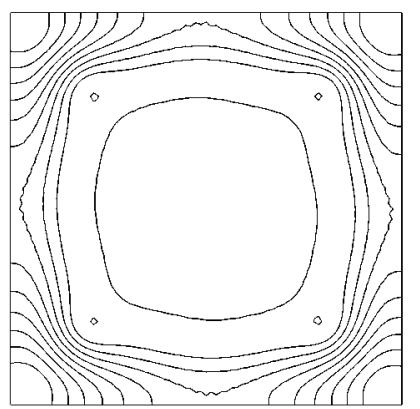

(c)

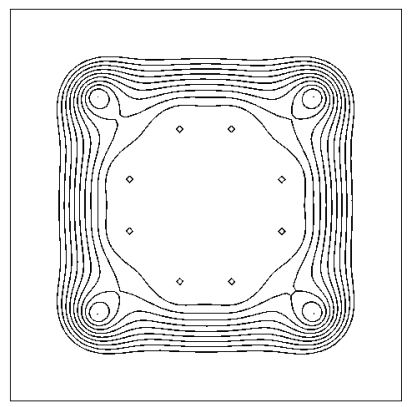

(b)

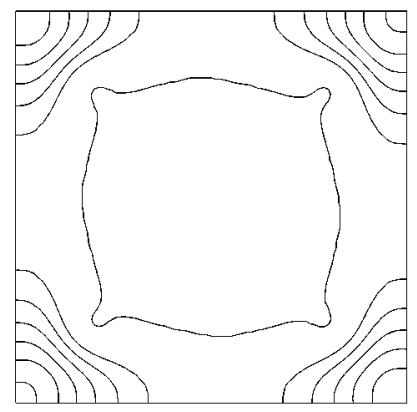

(d)

FIG. 4. Contour plots of $f \equiv|\psi|$ for an isotropic sample having a $10 \xi \times 10 \xi$ cross section with different magnetic-field strengths; $H / H_{c 2}=0.7$ (a), 1.0 (b), 1.6 (c), and 1.8 (d). For fields larger than $2.0 H_{c 2}$, the order parameter vanishes.

close to $H_{c 3}$. The predicted value of $H_{c 3}$ using the theory for semi-infinite samples ${ }^{1}$ is $1.695 H_{c 2}$. The observed value $H_{c 3}$ $\approx 2.0 H_{c 2}$ is higher than the predicted value. This is, of course, due to the finite size of the sample cross section and the corners.

Another series of computations was performed to observe the effects on the value of $H_{c 3}$ resulting from embedding the superconductor within a normal material other than a vacuum. (For the calculations reported on so far and for all other calculations given in the paper, the whole region exterior to the superconductor was assumed to be a vacuum.) These calculations were performed using the models and numerical techniques discussed in Ref. 26; see also Ref. 25. A series of calculations was performed on a cylindrical superconductor with a square cross section surrounded by normal material of different widths. For example, a $5 \xi \times 5 \xi$ superconducting sample was surrounded by a normal strip of width $5 \xi$ and a $9 \xi \times 9 \xi$ sample by a strip of width $\xi$. The region exterior to the normal material was assumed to be a vacuum. In all cases, for all applied fields above $H_{c 2}$, there was a total loss of superconductivity throughout the entire sample. As expected, our calculations supported the theory that $H_{c 3}$ is totally suppressed, i.e., $H_{c 3}=H_{c 2}$ in superconducting samples surrounded by normal materials.

\section{COMPUTATIONAL STUDY OF ANISOTROPIC SAMPLES}

The next example is for an anisotropic sample having a square cross section of size $10 \xi \times 10 \xi$. The $z$ axis is perpendicular to the sample cross section and the other two coordi-

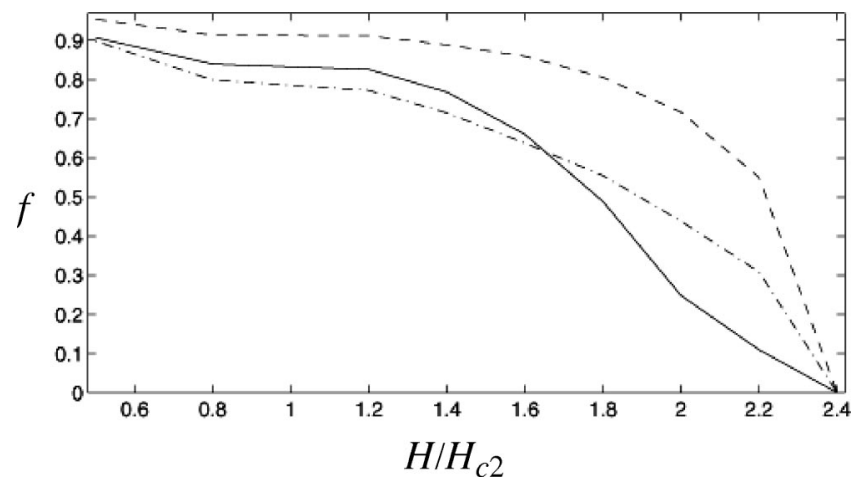

FIG. 5. $f \equiv|\psi|$ vs $H / \bar{H}_{c 2}$ for an anisotropic sample having a $10 \xi \times 10 \xi$ cross section and an in-plane mass ratio $3 / 2$. Solid curve: $f$ at the midsides, dashed curve: $f$ at the corners, and dot-dashed curve: average of $f$ over the sample perimeter.

nate axes are aligned with the boundary of the sample cross section. In this case, the mass tensor is diagonal. Specifically, we choose $m_{b}=m_{x x}=3 / 2, m_{c}=m_{y y}=1, m_{x y}=0$, and $m_{a}$ $=m_{z z}=2 / 3$. Note that for this anisotropic sample we have from Eq. (15) that $H_{c 2, z}=\bar{H}_{c 2} / \sqrt{m_{z z}}=\sqrt{3 / 2} \bar{H}_{c 2}$ so that the theoretically predicted value of the field at which superconductivity is completely suppressed in semi-infinite samples with this anisotropy is obtained from Eq. (17) to be $H_{c 3, z}$ $=1.695 H_{c 2, z}=2.076 \bar{H}_{c 2}$.

Now we turn to the results of computational simulations for the $10 \xi \times 10 \xi$ sample. The magnitude of the order parameter $f$ vs the applied field $H$ is given in Fig. 5. Again, the solid curve corresponds to $f$ at the midsides, the dashed curve to $f$ at the corners, and the dot-dashed curve to the average of $f$ over the sample perimeter. The midside values of $\psi$ again approach zero faster than the corner or average values. As predicted in Sec. III, the behavior of $f$ again conforms to a square-root-like behavior as the applied field is increased towards $H_{c 3}$. [We continue to scale $H$ with respect to $\bar{H}_{c 2}$ $=\phi_{0} / 2 \pi \xi^{2}$, where $\xi=\left(\xi_{a} \xi_{b} \xi_{b}\right)^{1 / 3}$, in order to make comparisons between isotropic and anisotropic calculations.] Due to the boundedness of the sample used for Fig. 5, we have that superconductivity is actually not completely suppressed until $H \approx 2.4 \bar{H}_{c 2}$.

Comparing the field values at which superconductivity is suppressed for the isotropic and anisotropic samples, one finds that their ratio is approximately $2 / 2.4=0.833$ which is very close to the ratio $\sqrt{m_{z z}}=\sqrt{2 / 3}=0.817$ predicted by the semi-infinite sample theory; see Eqs. (15) and (17). Thus, although the actual values of the field at which superconductivity is suppressed are affected by the presence of corners and the boundedness of the sample, the percentage change due to anisotropies is seemingly unaffected by these features.

In Fig. 6, contour plots of $f$ are shown for the steady states obtained with the applied magnetic field ranging from $0.5 \bar{H}_{c 2}$ to $2.2 \bar{H}_{c 2}$. For fields above $H_{c 2, z}=\sqrt{3 / 2} \bar{H}_{c 2}$, we see a combination of vortices and surface superconductivity. Due to the anisotropy, the vortices are now elliptical with the ratio of major to minor axes equal to $\sqrt{m_{x x} / m_{y y}}=\sqrt{3 / 2}$. Likewise, the thickness of the superconducting region near the surface of the sample is thicker adjacent to the sides parallel to the $x$ 


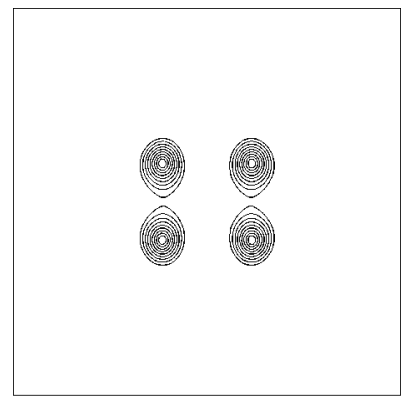

(a)

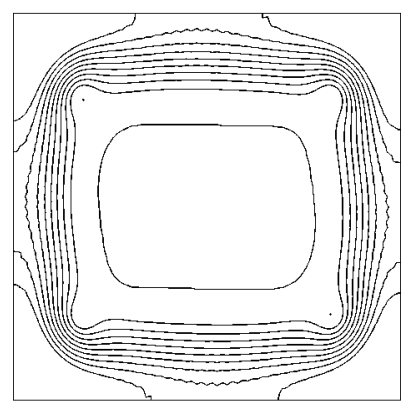

(c)

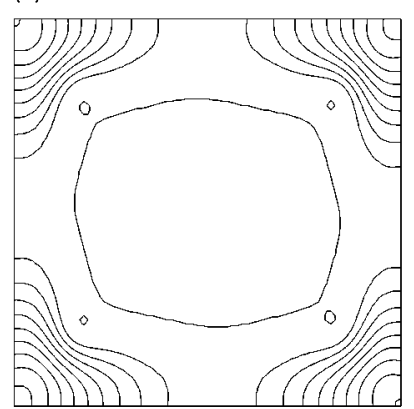

(e)

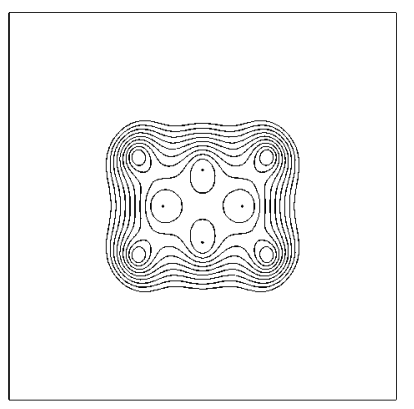

(b)

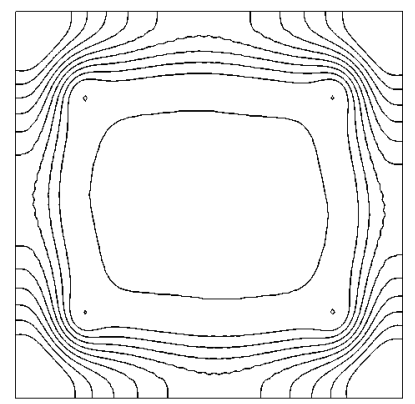

(d)

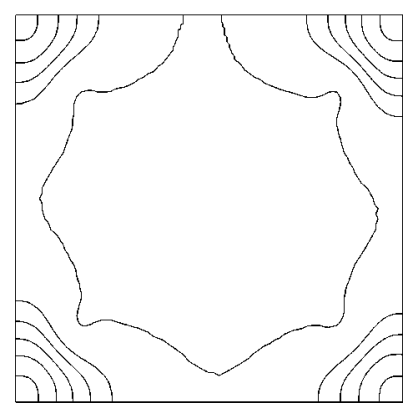

(f)

FIG. 6. Contour plots of $f \equiv|\psi|$ for an anisotropic sample having a $10 \xi \times 10 \xi$ cross section and mass ratio $3 / 2$ for $H / \bar{H}_{c 2}=0.5$ (a), 0.8 (b), 1.6 (c), 1.8 (c), 2.0 (e), and 2.2 (f); for fields larger than $2.4 \bar{H}_{c 2}$, the order parameter vanishes.

axis compared to the sides parallel to the $y$ axis; this is most easily seen by comparing the separation between contour lines. Again, these thicknesses are roughly in the ratio of $\sqrt{3 / 2}$ which is to be expected since this is the ratio of the coherence lengths in the directions perpendicular to the sides.

Note in Fig. 6(b) that in the contour plot corresponding to the field value $0.8 \bar{H}_{c 2}$, one can see several vortex cores near the center of the sample, and the ever present four vortices nearer to the corners. As the field increases, the former are no longer visible, while the latter persist until $H_{c 3, z}$ is reached.

The effects of anisotropy on the shape of the vortices and on the thickness of the surface superconductivity region are more visible if one increases the mass ratio. In Fig. 7, the contour plot on the left is for an isotropic sample having a cross section $5 \xi \times 5 \xi$ at a field strength of $1.8 H_{c 2}$. The one on the right is for an anisotropic sample having a cross section $5 \xi \times 5 \xi$ with masses $m_{x x}=6, m_{y y}=1, m_{z z}=1 / 6$, and $m_{x y}=0$ with a field strength of $3.8 \vec{H}_{c 2}$. Due to the larger

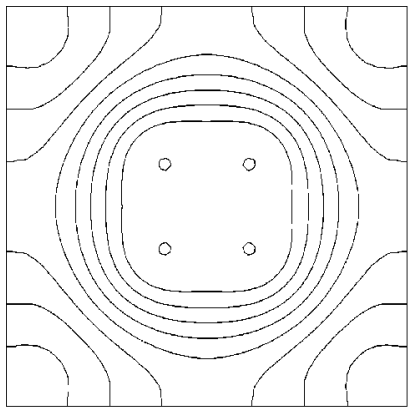

(a)

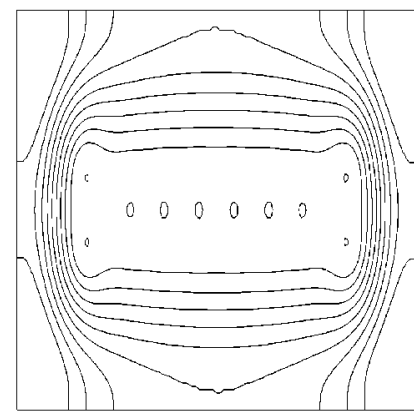

(b)
FIG. 7. Contour plots of $f \equiv|\psi|$ for isotropic (left) and anisotropic (right) samples of cross section $5 \xi \times 5 \xi$ with field strengths of $1.8 H_{c 2}$ and $3.8 \bar{H}_{c 2}$, respectively. The anisotropic sample has an in-plane mass ratio of 6 .

mass ratio, the effects of the anisotropy are now more clearly pronounced.

By the way, for these two samples, the calculations showed that superconductivity was completely suppressed for fields above $2.1 H_{c 2}$ for the isotropic sample and $5.1 \bar{H}_{c 2}$ for the anisotropic sample. The ratio $2.1 / 5.1=0.412$ is again in close agreement with the ratio $\sqrt{m_{z z}}=\sqrt{1 / 6}=0.408$ predicted by the theory for semi-infinite samples in this case for which we have $m_{z z}=1 / 6$ and $m_{x y}=0$; see Eqs. (15) and (17). Of course, due to the boundedness of the sample and the presence of corners, the actual values of these critical fields are larger than that predicted by the theory for semi-infinite samples.

\section{COMPUTATIONAL STUDY FOR CRYSTAL AXES OBLIQUE TO THE BOUNDARIES}

We now consider anisotropic samples for which the principal axes of the crystal are oblique to the boundaries of the sample. We will examine samples having rectangular cross sections aligned with the coordinate axes so that the crystal axes will not be parallel to the coordinate axes. We assume that one of the principal axes of the crystal, say the $a$ axis, is aligned with the $z$-coordinate axis and that the applied field is also in this direction. The $b$ axis of the lattice is assumed to form an angle $\theta$ with the $x$-coordinate axis which itself is parallel to a pair of sides of the rectangular sample. See Fig. 1 . We will refer to the case of $\theta=0$, i.e., the crystal axes are parallel to the sample boundaries, as the aligned case, and to any case for which $0<\theta<\pi / 2$ as an unaligned case.

We examine a rectangular sample having a cross section of size $30 \xi \times 10 \xi$; the larger length of two of the sides of this rectangle (compared to the previous calculations) will allow a reduction of the effects due to corners on at least part of the sample. The masses are chosen to be $m_{a}=m_{z z}=1 / 2, m_{b}$ $=2$, and $m_{c}=1$.

In Fig. 8, contour plots of $f$ are shown for the steady states obtained with the applied magnetic field ranging from $0.5 \bar{H}_{c 2}$ to $2.5 \bar{H}_{c 2}$. The figures on the left are for $\theta=0$; for those on the right, $\theta=\pi / 4$. As expected, the major and minor axes of the elliptically shaped vortices align themselves with the principal $b$ and $c$ axes of the crystal. The first thing to 


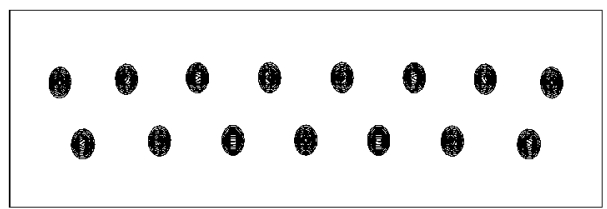

(a)

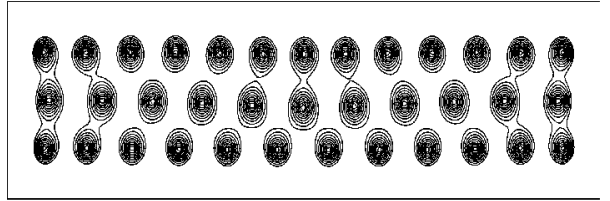

(c)

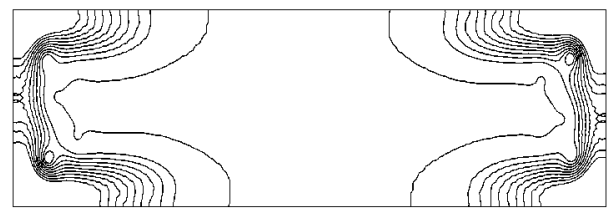

(e)

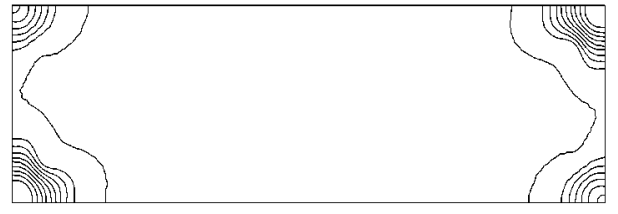

(g)

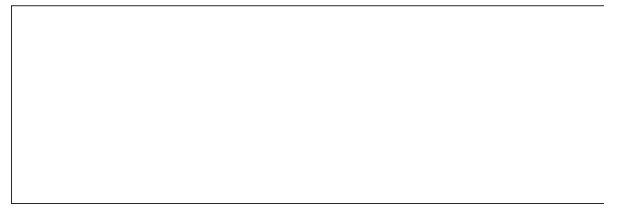

(i)

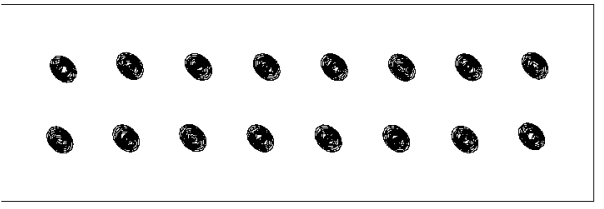

(b)

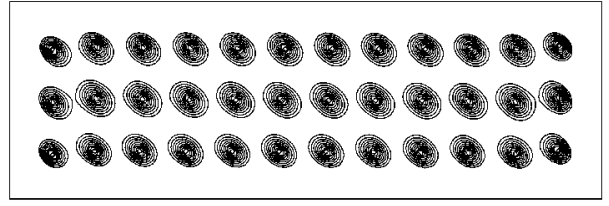

(d)

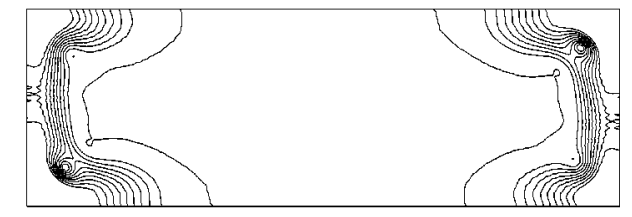

(f)

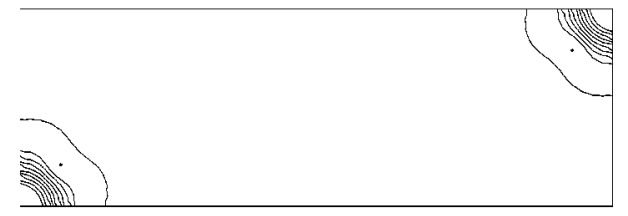

(h)

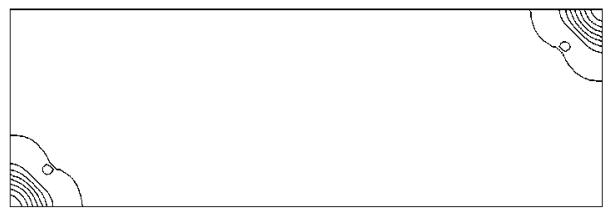

(j)
FIG. 8. Contour lines of $f$ for two $30 \xi \times 10 \xi$ anisotropic samples with in-plane mass ratio 2 . On the left, the crystal axes are aligned with the sample boundaries; on the right, the crystal axes are rotated by $45^{\circ}$. The applied field strengths are $H / \bar{H}_{c 2}=0.5$ [(a) and (b)], 1 [(c) and (d)], 1.5 [(e) and (f) $], 2[(\mathrm{~g})$ and $(\mathrm{h})]$, and $2.5[(\mathrm{i})$ and $(\mathrm{j})]$. notice is that the aligned sample $(\theta=0)$ becomes completely normal at a lower field than does the unaligned sample $(\theta$ $=\pi / 4$ ), i.e., the calculated value of $H_{c 3, a}$ for the first sample is lower than that for the second. On the other hand, the theory for semi-infinite samples predicts that, for the cases under study here, $H_{c 2, a}=\bar{H}_{c 2} / \sqrt{m_{z z}}=\sqrt{2} \bar{H}_{c 2}$ for both samples [see Eq. (15) with $m_{z z}=1 / 2$ ],$H_{c 3, a}=1.695 H_{c 2, a}$ $=2.397 \bar{H}_{c 2}$ for the sample having the crystal axes aligned with the boundaries [see Eq. (20) with $\theta=0$ ], and $H_{c 3, a}$ $=0.9428\left(1.695 H_{c 2, a}\right)=1.598 \bar{H}_{c 2, z}=2.260 H_{c 2}$ for the sample with $\theta=\pi / 4$ [see Eq. (20) with $\theta=\pi / 4$ ], i.e., superconductivity is completely suppressed at a higher field for the aligned sample than for the unaligned one.

An examination of Fig. 8 reveals that superconductivity persists for higher fields for the unaligned sample at the corners. At the midsides of the samples we would expect that the effects due to the corners are somewhat mitigated. Indeed, there is an indication from that figure that superconductivity persists for higher fields at the midsides for the aligned sample; see Fig. 8(e) and Fig. 8(f) for the long sides and Fig. 8(g) and Fig. 8(h) for the short sides. This is verifed in Fig. 9 where the values of $f$ at the middle of the long sides vs the applied field are given for both samples. It is clear that superconductivity is suppressed at a lower value of the field for the unaligned sample. In fact, from Fig. 9, the ratio of the values of the field at which superconductivity is suppressed for the unaligned case with $\theta=\pi / 4$ and the aligned case with $\theta=0$ is roughly 0.94 which is in excellent agreement with the theoretically predicted value $2.260 / 2.397=0.93$. Of course, the samples used here are extremely small, so that the effects of corners are much more pronounced than they

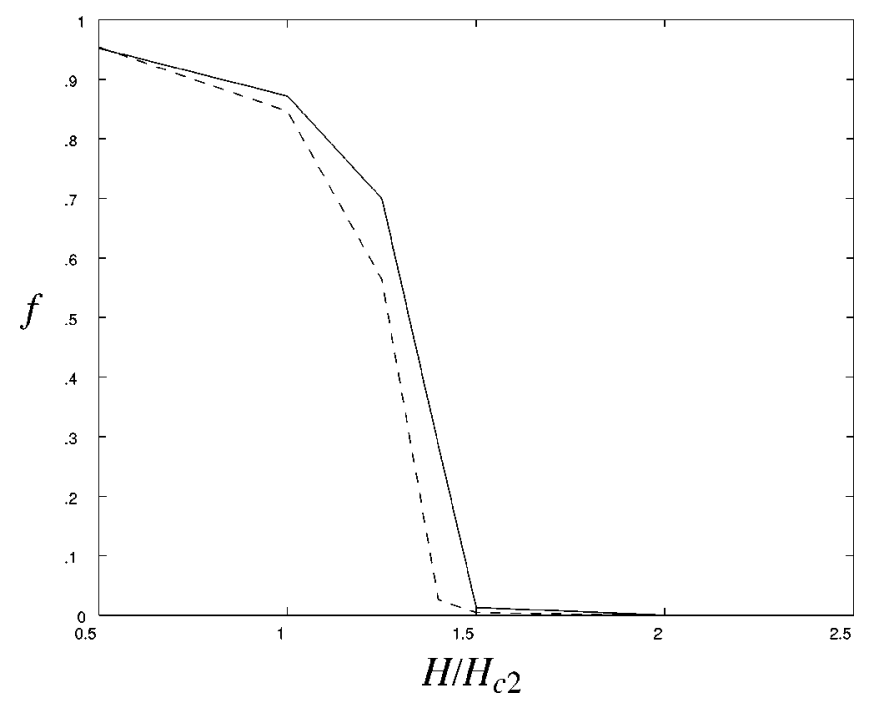

FIG. 9. Value of $f$ at the middle of the long sides of the aligned (solid) and unaligned (dashed) samples. 
would be for more realistic sample sizes.

For the above example, $H_{c 3, a}>H_{c 2, a}$ for all angles $\theta$, so that the surface superconductivity state is present for all angles. Calculations were also performed for an anisotropic sample of a cross section $10 \xi \times 10 \xi$ having masses $m_{a}$ $=1 / 10, m_{b}=10$, and $m_{c}=1$. If the sample were semi-infinite in extent, according to Eq. (21), the surface superconductivity state should not be possible for $37.04^{\circ} \leqslant \theta \leqslant 52.96^{\circ}$, and in particular, for $\theta=45^{\circ}$. However, we found that due to the effect of corners for this small sample, the surface superconductivity state persisted even for $\theta=45^{\circ}$ for fields as large as $5 \bar{H}_{c 2}$. Note that in this case $H_{c 2, a}=3.162 \bar{H}_{c 2}$ and, for the semi-infinite case, one obtains the theoretical value of $H_{c 3, a}$ $=0.9746 H_{c 2, a}=3.082 \bar{H}_{c 2}$.

We note that the lack of symmetry in plots such as Fig. 8(e) and Fig. 8(g) is a residual of the fact that for lower fields, e.g., see Fig. 8(a), it is not possible to fit a symmetric arrangement of vortices into the rectangular sample. Of course, the lack of alignment between the sample boundaries and the crystal axes whenever $\theta \neq 0$ induces the lack of symmetry seen for the plots in the right-hand column of Fig. 8.

\section{CONCLUDING REMARKS}

For isotropic, semi-infinite samples, the critical field at which superconductivity is completely suppressed was deter- mined by Saint-James and de Gennes. ${ }^{1}$ We have extended that theory to the case of anisotropic superconductors having one crystal axis parallel to the surface of the superconductor and the other two crystal axes arbitrarily oriented with respect to that surface. We have also examined the dependence of the order parameter on the applied field for fields near but below the critical field. A number of results of computational simulations were presented which both illustrate the theoretical results and the effects of corners and finite-sized samples on the critical field. These studies indicate that, due to the finite size of the sample cross section and due to corners, the surface superconductivity state persists for fields above the predicted theoretical value for semi-infinite samples. The computational studies also show that vortices exist within the surface superconductivity sheath for fields above the bulk critical field.

\section{ACKNOWLEDGMENTS}

Ames Laboratory is operated for the U.S. Department of Energy by Iowa State University under Contract No. W-7405-Eng-82. This research was supported by the Director for Energy Research, Office of Basic Energy Sciences.
${ }^{1}$ D. Saint-James and P. de Gennes, Phys. Lett. 7, 306 (1963).

${ }^{2}$ A. Houghton and F. McLean, Phys. Lett. 19, 172 (1965).

${ }^{3}$ H. Fink, Phys. Rev. 177, 1017 (1969).

${ }^{4}$ M. Millman and J. Keller, J. Math. Phys. 10, 342 (1969).

${ }^{5}$ A. Mel'nikov, Mod. Phys. Lett. B 19, 1211 (1990).

${ }^{6}$ S. Chapman, Eur. J. Appl. Math 5, 449 (1994).

${ }^{7}$ P. Bauman, D. Phillips, and Q. Tang, Arch. Ration. Mech. Anal. 142, 1 (1998).

${ }^{8}$ A. Bernoff and P. Sternberg, J. Math. Phys. 39, 1272 (1998).

${ }^{9}$ K. Lu and X. Pan, J. Math. Phys. 40, 2647 (1999).

${ }^{10}$ M. Del Pino, P. Felmer, and P. Sternberg, Commun. Math. Phys. 210, 413 (2000).

${ }^{11}$ K. Lu and X. Pan, Trans. Am. Math. Soc. 352, 1247 (2000).

${ }^{12}$ H. Jadallah, J. Math. Phys. 42, 4101 (2001).

${ }^{13}$ Y. Almog, J. Math. Phys. 41, 7889 (2001).

${ }^{14}$ O. Buisson, P. Gandit, R. Rammal, Y. Wang, and B. Pannetier, Phys. Lett. A 150, 36 (1990).

${ }^{15}$ V. Moshchalkov, L. Gielen, C. Stunk, R. Jonckheere, X. Qin, C. Van Haesendock, and Y. Bruynseraede, Nature (London) 373, 319 (1995).
${ }^{16}$ V. Bruyndoncx, J. Rodrigo, T. Pog, L. Van Look, and V. Moshchalkov, Phys. Rev. B 60, 4285 (1999).

${ }^{17}$ R. Kato, Y. Enomoto, and S. Maekawa, Phys. Rev. B 47, 8016 (1993).

${ }^{18}$ H. Jadallah, J. Rubinstein, and P. Sternberg, Phys. Rev. Lett. 82, 2935 (1999).

${ }^{19}$ V. Schweigert and F. Peeters, Phys. Rev. B 60, 3084 (1999).

${ }^{20}$ F. Brosens, J. Devreese, and V. Fomin, Solid State Commun. 111, 565 (1999).

${ }^{21}$ A. Abrikosov, Fundamentals of the Theory of Metals (NorthHolland, Amsterdam, 1988).

${ }^{22}$ V. Kogan and J. Clem, Phys. Rev. B 24, 2497 (1981).

${ }^{23}$ V. Klemm and J. Clem, Phys. Rev. B 21, 1868 (1980).

${ }^{24}$ Q. Du, M. Gunzburger, and J. Peterson, Phys. Rev. B 46, 9027 (1992).

${ }^{25}$ Q. Du, M. Gunzburger, and J. Peterson, Phys. Rev. B 51, 16194 (1995).

${ }^{26}$ J. Chapman, Q. Du, and M. Gunzburger, Eur. J. Appl. Math 6, 97 (1995). 